\title{
Advanced FIB-based Preparation of Cryogenically-prepared Specimens for APT Analysis
}

Daniel E. Perea ${ }^{1}$, Daniel K. Schreiber ${ }^{2}$, Mark Wirth ${ }^{1}$, James E. Evans ${ }^{1}$

1. Earth and Biological Sciences Directorate, Pacific Northwest National Laboratory, Richland, WA USA.

2. Energy and Environment Directorate, Pacific Northwest National Laboratory, Richland, WA USA.

* Corresponding author: daniel.perea@pnnl.gov

The utilization of the focused ion beam scanning electron microscope (FIB-SEM) has aided the application of Atom Probe Tomography (APT) to a continually-growing variety of materials, including soft biological materials [1]. However, the regular application of APT to hydrated interfaces such as those found in biological and environmental materials is lacking in large part due to difficulties in specimen preparation under high and ultra-high vacuum conditions. Although a FIB-SEM based cryogenic specimen preparation approach offers a rational route to the preparation of such specimens for APT analysis, many practical aspects such as welding and limitations in the cryo stage manipulation makes it challenging for routine liftout of site-targeted specimens. Here we describe our recent efforts in the development of a sitespecific liftout and attachment scheme for cryogenically cooled specimens involving a combination of redeposition and overcoating of organic and organometallic molecules, as shown in Fig. 1a-d for a cryogenically-frozen corroded glass sample containing a hydrated porous gel surface layer [2]. Application of an annular milling pattern to the frozen specimens leads to a needle-shaped morphology necessary for APT analysis with the targeted water-ice filled porous corroded silicate glass region of interest near the apex (Fig. 1e). Using a custom shuttle device and environmental transfer hub at PNNL to facilitate environmentally-protected specimen transfer [3], APT analysis was applied to directly map, for the first time, a water/solid interface in 3D to reveal the complex nanoscale water-filled porous network of corroded glass (Fig. 1f). Application of this unique specimen preparation approach to biological specimens will also be discussed, as well as the application of a unique cryo nanomaniupulator with integrated gas injection system to enable controlled FIB-based welding under cryogenic temperatures.

\section{References:}

[1] DE Perea, J Liu, J Bartrand, Q Dicken, ST Thevuthasan, ND Browning, JE Evans. Scientific Reports 6 (2016), p. 22321.

[2] DK Schreiber, DE Perea, JV Ryan, JE Evans, JD Vienna, Ultramicroscopy 194 (2018), p. 89.

[3] D.E. Perea, SSA Gerstl, J Chin, B Hirschi, JE Evans, Advanced Structural and Chemical Imaging 3 (2017), p. 12.

[4] The research was performed using EMSL (grid.436923.9), a DOE Office of Science User Facility sponsored by the Office of Biological and Environmental Research. 
Attachment of frozen hydrated corroded glass to APT micropost using cryo FIB
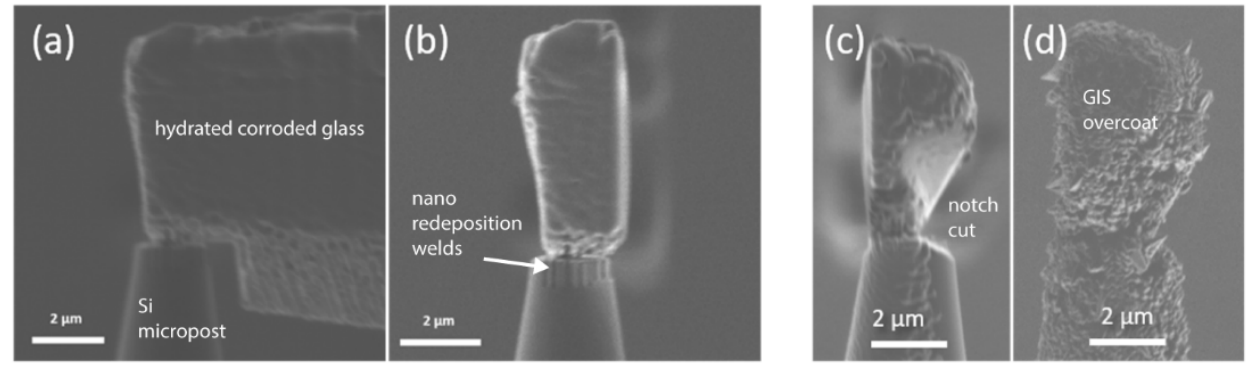

(e)

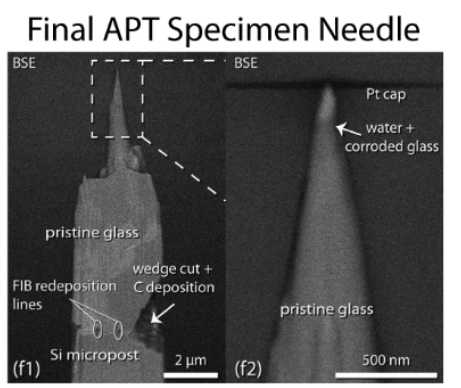

(f) APT reconstruction

(f) of water-filled porous glass

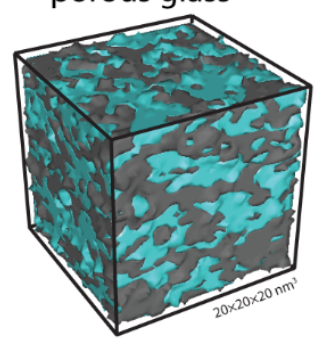

Figure 1. (a) placement and (b) attachment of a portion of cryogenically frozen hydrated corroded glass to $\mathrm{Si}$ micropost using nanoscale redeposition welds. To better secure the frozen specimen to the Si micropost, a (c) notch cut was cut on the side and (d) overcoated with $\mathrm{C}$ or Pt from the gas injection system (GIS) of the FIB. (e) Final specimen needle shape following an annular milling pattern. Note both the Pt GIS overcoat cap and water+corroded glass layer are visible near the apex as highlighted. (f) 3D isoconcentration surface showing water filled (blue) porous silicate glass (grey) of the corroded glass layer. 